\title{
Characterization of cubosomes as a targeted and sustained transdermal delivery system for capsaicin
}

This article was published in the following Dove Press journal:

Drug Design, Development and Therapy

3 August 2015

Number of times this article has been viewed

\author{
Xinsheng Pengl,* \\ Yanfang Zhoul,* \\ $\mathrm{Ke} \mathrm{Han}{ }^{2,3}$ \\ Lingzhen Qin ${ }^{3}$ \\ Linghui Dian' \\ $\mathrm{Ge} \mathrm{Li}{ }^{4}$ \\ Xin $\operatorname{Pan}^{3}$ \\ Chuanbin $\mathrm{Wu}^{3}$
}

'Guangdong Medical University, Dongguan, ${ }^{2}$ The Second Affiliated Hospital of Guangzhou Medical University, ${ }^{3} \mathrm{School}$ of Pharmaceutical Sciences, Sun Yat-Sen University, ${ }^{4}$ Guangzhou Neworld Pharmaceuticals Co. Ltd., Guangzhou, Guangdong, People's Republic of China

*These authors contributed equally to this work

\footnotetext{
Correspondence: Xin Pan; Chuanbin Wu School of Pharmaceutical Sciences, Sun Yat-Sen University, University Town, Guangzhou 510006, Guangdong, People's Republic of China Tel +86 2039943 II I7 Fax +86 $2039943115 ;+862039943120$ Email pxin_1385@।63.com; chuanbin_wu@I26.com
}

\begin{abstract}
Phytantriol- and glycerol monooleate-based cubosomes were produced and characterized as a targeted and sustained transdermal delivery system for capsaicin. The cubosomes were prepared by emulsification and homogenization of phytantriol (F1), glycerol monooleate (F2), and poloxamer dispersions, characterized for morphology and particle size distribution by transmission electron microscope and photon correlation spectroscopy. Their Im3m crystallographic space group was confirmed by small-angle X-ray scattering. An in vitro release study showed that the cubosomes provided a sustained release system for capsaicin. An in vitro diffusion study conducted using Franz diffusion cells indicated that the skin retention of capsaicin from cubosomes in the stratum corneum was much higher $(2.75 \pm 0.22 \mu \mathrm{g}$ versus $4.32 \pm 0.13 \mu \mathrm{g}$, respectively) than that of capsaicin cream $(0.72 \pm 0.13 \mu \mathrm{g})$. The stress testing showed that the cubosome formulations were stable under strong light and high temperature for up to 10 days. After multiapplications on mouse skin, the irritation of capsaicin cubosomes and cream was light with the least amount of side effects. Overall, the present study demonstrated that cubosomes may be a suitable skin-targeted and sustained delivery system for the transdermal administration of capsaicin.
\end{abstract}

Keywords: cubosomes, skin-targeted delivery, capsaicin

\section{Introduction}

Capsaicin (trans-8-methyl- $N$-vanillyl-6-nonenamide; Figure 1) is a natural alkaloid extracted from the fruits of the capsicum plant family. It exhibits broad bioactivities and has been used for the treatment of psoriasis, pruritus, apocrine chromhidrosis, and contact allergy. ${ }^{1,2}$ Because of its high degree of first-pass metabolism in the gastrointestinal tract and short half-life by intravenous administration (about 7 minutes), ${ }^{3}$ the transdermal delivery system seems to be more advantageous for capsaicin. Hydrogel, solution, ointment and cream, microemulsion, transfersome, noisome, and liposome preparations of capsaicin have been documented in the literature to treat pain-related disorders such as rheumatoid arthritis, osteoarthritis, diabetic neuropathy, and postoperative pain..$^{4-9}$ Among them, transfersomes, niosomes, and liposomes are especially efficient to enhance the penetration of capsaicin through the skin and the absorption of the drug into the systemic circulation. Due to its short half-life, the transdermal application of capsaicin needs more frequent replacements in order to achieve satisfactory pain relief. However, the replacement of postoperation or postincision wound dressing is painful. In addition, overdosage of capsaicin adsorbed by systemic circulation may induce potential systemic side effects. So, skin-targeted delivery would be optimal for capsaicin, which could ensure that the drug would be mainly absorbed by the skin with less systemic absorption as compared with the conventional transdermal application. ${ }^{10-12}$ 


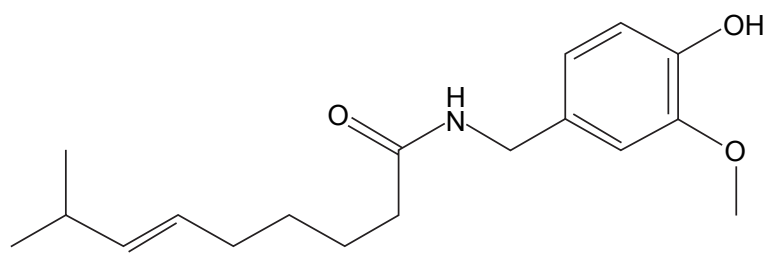

Figure I Chemical structure of capsaicin.

Therefore, the purpose of this study was to develop a novel delivery system that could enhance drug absorption to the skin and, at the same time, retard the systemic diffusion of the skin-absorbed drug.

The cubic phase is an intriguing drug delivery system consisting of a curved continuous lipid bilayer extending in three dimensions and separating two congruent networks of water channels. ${ }^{13-16}$ It can enclose hydrophilic, amphiphilic, and hydrophobic substances ranging from small-molecularweight drugs to proteins, peptides, amino acids, and nucleic acids. ${ }^{16}$ Typically, three forms of cubic phase have been used as drug delivery systems: cubic phase gel; cubic phase precursor; and cubosome. Cubic phase gels have been commonly used for mucosal, vaginal, periodontal, and transdermal drug delivery. Bender et $\mathrm{al}^{17}$ reported that lipid cubic phase gels significantly increased the delivery of $\delta$-aminolevulinic acid hydrochloride and methyl $\delta$-aminolevulinate hydrochloride through topical administration as compared with the normal ointments. However, the stiff and viscous nature of the cubic phase gel limits its potential use. ${ }^{15,16}$ Though low viscous cubic phase gel could be obtained by adding organic solvents (propylene glycol, ethanol, polyethylene glycol, and $N$-methyl-2-pyrrolidone), ${ }^{18,19}$ we found that the gel was not easy to spread and adhere to the skin. Cubosomes posses much lower viscosity than the bulk cubic phase, ${ }^{16}$ and they show better storing stability at room temperature and greater endurance of heat treatment as compared to liposomes. ${ }^{20-22}$ Besides, cubosomes could exist at almost any dilution level in water, and drug leakage was less of a concern as compared with liposomes.
Cubosomes and liposomes both have a bilipid membrane, and liposome gel could act in the skin-targeted transdermal drug delivery of hydrocortisone. ${ }^{23}$ Gan et $\mathrm{al}^{24}$ reported that dexamethasone-loaded cubosomes exhibited a permeability coefficient that was 4.5-fold greater than eye drops. Also, Morsi et $\mathrm{al}^{25}$ successfully developed silver sulfadiazineloaded cubosome hydrogels for the topical treatment of burns with better patient compliance, excellent healing results, and the least amount of side effects. All of these properties are favorable for capsaicin delivery. Therefore, we hypothesized that cubosomes with such favorable properties might represent a promising vehicle for the effective transdermal delivery of capsaicin. As such, we performed the following experiments to test our hypothesis.

\section{Materials and methods Materials and reagents}

Glycerol monooleate (GMO) (DIMODAN ${ }^{\circledR}$ MO/D KOSHER; catalog number: 116703) was kindly provided by Danisco Cultor (Brabrand, Denmark) and used as received. Poloxamer $407\left(\mathrm{PEO}_{98} \mathrm{POP}_{67} \mathrm{PEO}_{98}\right)$ was a gift from BASF (Ludwigshafen, Germany). Phytantriol (PYT) was purchased from DSM (Basel, Switzerland). Capsaicin was purchased from Wuhan Hengshuo Technology Development Co. Ltd. (Wuhan, Hubei, People's Republic of China). Capsaicin cream (Changchun Puhua Pharmaceutical Co., Ltd., Changchun, Jilin, People's Republic of China) contained $2.5 \mathrm{mg} / 10 \mathrm{~g}$ of capsaicin. A dialysis bag with a molecular weight cutoff of 14,000 Da (Viskase Companies, Inc., Darien, IL, USA) was used. Milli-Q grade water purified through a Millipore system (ELGA LabWater, Sartorius; Reading, UK) was used throughout this study. All other reagents were of high-performance liquid chromatography (HPLC) or analytical grade and used as received. Animal experiments were carried out according to the protocol approved by the Experimental Animal Committee of Guangdong Medical University, Dongguan, Guangdong, People's Republic of China.

Table I Composition of prepared cubosomes

\begin{tabular}{lllllll}
\hline Formulation & $\begin{array}{l}\text { Capsaicin } \\
(\mathbf{m g})\end{array}$ & FI27 $(\mathbf{g})$ & PYT $(\mathbf{g})$ & GMO $(\mathbf{g})$ & Water & $\begin{array}{l}\text { Capsaicin } \\
\text { concentration }(\mu g / m L)\end{array}$ \\
\hline FI & 10.88 & 0.3047 & 3.0096 & 0 & Adequate & 250 \\
F2 & 10.30 & 0.3028 & 0 & 3.0035 & Adequate & 250 \\
\hline
\end{tabular}

Notes: FI, PYT based cubosomes; F2, GMO based cubosomes. The concentration of capsaicin in both cubosomes are $250 \mu \mathrm{g} / \mathrm{mL}$. The cubosomes are prepared as follows: GMO or PYT and FI27 were first completely melted and then capsaicin was added to dissolve. $6.7 \mathrm{~mL}$ water was then added gradually and vortex-mixed to form the cubic phase gel after equilibration for 48 hours at room temperature. The gel was disrupted under mechanical stirring with addition of approximately $20 \mathrm{~mL}$ water. Subsequently, the crude dispersion of the gel was fragmented for 10 minutes by intermittent probe sonication under cooling in a $20^{\circ} \mathrm{C}$ water bath. Afterwards, the resulting milky coarse dispersion was homogenized using a high pressure homogenizer to form cubosome nanoparticles.

Abbreviations: PYT, phytantriol; GMO, glycerol monooleate; FI27, Poloxamer 407. 


\section{Preparation of capsaicin-loaded cubosomes}

Capsaicin-loaded cubosomes were prepared through the fragmentation of GMO or PYT/poloxamer 407 bulk cubic phase gels. The formulations are shown in Table 1. GMO or PYT and poloxamer 407 were first completely melted at $60^{\circ} \mathrm{C}$ in a hot water bath, and then capsaicin was added to dissolve/ blend under continuous stirring. Water $(\sim 6.7 \mathrm{~mL})$ was then added gradually and the mixture was vortex-mixed to achieve a homogeneous state. After equilibration for 48 hours at room temperature, the cubic phase gel was formed. With the addition of approximately $20 \mathrm{~mL}$ of water, the cubic gel was disrupted under mechanical stirring. Subsequently, the crude dispersion of the gel was fragmented for 10 minutes by intermittent probe sonication (JYD-650; Shanghai Zhixin Instrument Co., Ltd., Shanghai, People's Republic of China) at $400 \mathrm{~W}$ energy input with a pulse mode (9-second pulses interrupted by 18 -second breaks) under cooling in a $20^{\circ} \mathrm{C}$ water bath. Afterward, the resulting milky coarse dispersion was homogenized using a high-pressure homogenizer (Em-C3; AVESTIN, Inc., Ottawa, ON, Canada) at certain high pressures and cycles to form an opalescent dispersion of the cubic nanoparticles. The final dispersion of liquid crystal nanoparticles was stored at room temperature for further studies. ${ }^{26}$

\section{Determination of particle size of cubosomes}

The size distribution of cubosomes was measured using a Zetasizer Nano ZS90 (Malvern Instruments, Malvern, UK). The samples were diluted 50 -fold with water to $2 \%$ before measurement. Cubosomal size was analyzed by the Dispersion Technology Software provided by Malvern Instruments.

\section{Transmission electron microscopy}

Transmission electron microscopy (TEM) images were taken using a JEM 1400 JEOL instrument (JEOL, Tokyo, Japan). Specimens were prepared by drop casting of the cubosomes $(10 \mu \mathrm{L})$ on a copper grid with $10 \%$ phosphotungstic acid. The size distribution was determined by examining an overview TEM image using software.

\section{Small-angle X-ray scattering}

Small-angle X-ray scattering (SAXS) measurements were carried out on a high-flux SAXS instrument (Anton Paar $\mathrm{GmbH}$, Graz, Austria) operating in the line of collimation and equipped with an imaging plate (IP) as a detector. The IP with a pixel size of $42.3 \times 42.3 \mu \mathrm{m}^{2}$ was extended into a wideangle range (the q range covered by the IP was up to $28 \mathrm{~nm}^{-1}$, $\mathrm{q}=[4 \pi \sin \theta] / \lambda$, where $\lambda$ is the wavelength of $0.1542 \mathrm{~nm}$ and $2 \theta$ is the scattering angle). The liquid samples were carefully loaded into a quartz capillary with a diameter of $1 \mathrm{~mm}$ and exposed for 60 minutes.

\section{Encapsulation efficiency}

Encapsulation efficiency (EE) was evaluated using a membrane dialysis method, as described. ${ }^{27}$ The sample $(2 \mathrm{~mL})$ was dispersed in a dialysis bag, which was placed into a flask and filled with $50 \mathrm{~mL}$ of water. The flask was then put in a rotary incubator shaker with a setting of $100 \mathrm{rpm}$ and $37^{\circ} \mathrm{C}$. After 4 hours of shaking, the concentration of free capsaicin was determined by HPLC. ${ }^{28}$ The EE was calculated per the formula:

$$
\mathrm{EE} \%=\left(\mathrm{C}_{\text {total con }}-\mathrm{C}_{\text {free con }}\right) / \mathrm{C}_{\text {total con }} \times 100 \%
$$

\section{Stability study}

The physical stability of the cubosomes was studied by stress testing according Chinese Pharmacopoeia 2010 (part 2, Appendix XIX C). Namely, the cubosomal samples were deposited and sealed in glass sterile vials, and then the vials were placed in a drug stability test chamber (Chongqing Yongsheng Experiment Instrument Factory, Chongqing, People's Republic of China) under strong light $(4,500 \pm 500$ lx) and high temperature $\left(60^{\circ} \mathrm{C}\right)$, respectively, for 10 days. At the days 5 and 10, the appearance and particle size of the samples were checked.

\section{In vitro release and evaluation of the release mechanism}

The release of capsaicin from cubosomes in water at $37^{\circ} \mathrm{C}$ was evaluated by the dialysis method. The cubosomal sample $(5 \mathrm{~mL})$ was transferred into a dialysis bag, which was placed into a flask and filled with $250 \mathrm{~mL}$ of water. The flask was then put into a rotary incubator shaker at $50 \mathrm{rpm}$ and $37^{\circ} \mathrm{C}$. An aliquot of $5 \mathrm{~mL}$ was taken periodically and replaced with the same volume of water. The concentration of the released capsaicin was determined by HPLC on the basis of the standard curve. Different mathematical models of Higuchi, first-order, zero-order, Weibull, Hixson-Crowell, and Ritger-Peppas equations were used to explain the mechanism of capsaicin release from the cubosomes (data are shown in Table 2)..$^{29,30}$

\section{In vitro percutaneous permeation}

In vitro permeation procedures of the capsaicin-contained cubosome application were determined using vertical Franz diffusion 
Table 2 Fitting of drug release data according to various equations

\begin{tabular}{|c|c|c|c|c|}
\hline \multirow[t]{2}{*}{ Model } & \multirow[t]{2}{*}{ Cubosomes } & \multirow[t]{2}{*}{ Fitting equation } & \multicolumn{2}{|c|}{$\begin{array}{l}\text { Fitting assessment } \\
\text { parameters }\end{array}$} \\
\hline & & & $R^{2}$ & $\mathbf{K}$ \\
\hline \multirow[t]{2}{*}{ Higuchi equation } & $\mathrm{FI}$ & $\mathrm{Q}=7.236 \mid t^{1 / 2}-2.2083$ & 0.9973 & 7.2361 \\
\hline & $\mathrm{F} 2$ & $\mathrm{Q}=4.3616 t^{1 / 2}-0.3376$ & 0.9951 & 4.3616 \\
\hline \multirow[t]{2}{*}{ First-order equation } & $\mathrm{FI}$ & $\ln (100-Q)=-0.0134 t+4.5865$ & 0.984 & -0.0134 \\
\hline & F2 & $\ln (100-Q)=-0.0061 t+4.5305$ & 0.9382 & $-0.006 \mid$ \\
\hline \multirow[t]{2}{*}{ Zero-order equation } & $\mathrm{FI}$ & $\mathrm{Q}=1.0034 t+8.463$ & 0.9615 & 1.0034 \\
\hline & F2 & $\mathrm{Q}=0.4855 t+7.6396$ & 0.9713 & 0.4855 \\
\hline \multirow[t]{2}{*}{ Weibull equation } & $\mathrm{FI}$ & $\ln [\ln 100 /(100-Q)]=0.6233 t-2.8947$ & 0.9951 & 0.6233 \\
\hline & $\mathrm{F} 2$ & $\ln [\ln (100 /(100-Q))]=0.5448 t-3.1796$ & 0.9885 & 0.5448 \\
\hline \multirow[t]{2}{*}{ Hixson-Crowell equation } & $\mathrm{FI}$ & $(100-Q)^{1 / 3}=-0.0188 t+4.5159$ & 0.9752 & -0.0188 \\
\hline & F2 & $(100-Q)^{1 / 3}=-0.0087 t+4.5248$ & 0.953 & -0.0087 \\
\hline \multirow[t]{2}{*}{ Ritger-Peppas equation } & $\mathrm{FI}$ & $\ln Q=0.5605 \ln t+1.7185$ & 0.9966 & 0.5605 \\
\hline & F2 & $\ln Q=0.5036 \ln t+1.4333$ & 0.9902 & 0.5036 \\
\hline
\end{tabular}

Notes: FI, PYT based cubosomes; F2, GMO based cubosomes. The concentration of capsaicin in both cubosomes are $250 \mu \mathrm{g} / \mathrm{mL}$.

Abbreviations: Q, cumulative capsaicin released from cubosomes (\%); t, time (hours); K, release rate constants; PYT, phytantriol; GMO, glycerol monooleate.

cells with the epidermis facing the donor compartment. The abdominal skin of excised Sprague Dawley rats was used as the model membrane after the subcutaneous fat was maximally removed and the hair was shaved by an electric clipper.

The test formulations ( $1 \mathrm{~g}$ in $1 \mathrm{~mL})$ were applied on the diffusion surface of the skin $\left(2.8 \mathrm{~cm}^{2}\right.$ available diffusion area) in the donor compartment. The receiver compartment was filled with $6.5 \pm 0.5 \mathrm{~mL}$ of $20 \%$ ethanol solution, stirred at $300 \mathrm{rpm}$, and maintained at $32^{\circ} \mathrm{C} \pm 0.5^{\circ} \mathrm{C}$ using a circulating water bath. Ethanol was added to increase the solubility of capsaicin to reach the sink condition. Skin permeation studies were performed for 24 hours under unocclusive conditions, and $1 \mathrm{~mL}$ of the samples were withdrawn from the receptor at predetermined intervals and immediately replaced by an equal volume of fresh receptor solution. The samples were assayed by HPLC, as described previously $(20 \mu \mathrm{L}$ of the sample was injected into a Kromasil $\mathrm{C}_{18}$ at room temperature). The mobile phase was methanol:water $=70: 30$ with a flow rate of $1.0 \mathrm{~mL} / \mathrm{minute}$; the detection wavelength was $280 \mathrm{~nm} \cdot{ }^{28}$ Each sample was performed in triplicate. Cumulative permeated amounts were plotted against time. The permeation rate of capsaicin at steady state,

$$
\left(\mathrm{Js}=\mathrm{dQ} / \mathrm{dt}, \mu \mathrm{g} \cdot \mathrm{cm}^{-2} \cdot \mathrm{hour}^{-1}\right)
$$

through the rat skin was calculated by linear regression..$^{31,32}$

\section{Skin retention of capsaicin}

The skin retention of capsaicin was determined as described previously. ${ }^{33}$ At 24 hours postapplication, the treated skin surfaces were thoroughly washed with distilled water and wiped with cotton swabs to remove the excess formulation. To separate the stratum corneum (SC) from the remaining epidermis (E) and dermis (D), the skin sections were subject to tape stripping. The skin was stripped with 15 pieces of adhesive tape; the first one was discarded, and the other tapes containing SC were immersed in $5 \mathrm{~mL}$ of methanol, vortex stirred for 2 minutes, and sonicated with probe sonication for 10 minutes. The remaining $(\mathrm{E}+\mathrm{D})$ was cut into small pieces, vortex stirred for 2 minutes in $10 \mathrm{~mL}$ of methanol, followed by probe sonication for 10 minutes. All samples were centrifuged at 15,000 rpm for 10 minutes; the supernatant was collected and the extract was analyzed for capsaicin content using HPLC.

\section{Evaluation of skin irritation}

Histological changes in Kunming mouse skin were examined after treatment with different capsaicin applications for 7 days to evaluate the possible skin irritation caused by the formulations. Capsaicin formulations (cubosomes, cream; $500 \mathrm{~mL}$ each) were applied topically on a limited area $\left(2 \times 2 \mathrm{~cm}^{2}\right)$ of skin on the back of the mouse twice a day for 1 week. On the 7 th day, the mice were killed and the treated skin area was dissected. After fixation, the skin samples were dehydrated and embedded in paraffin, and then $5 \mu \mathrm{m}$ sections of skin were stained with hematoxylin and eosin for observation under microscope. The skin of the untreated mouse was used as the control.

\section{Statistical analysis}

All experimental data were expressed as the mean \pm standard deviation. Statistical analysis was performed using SPSS 


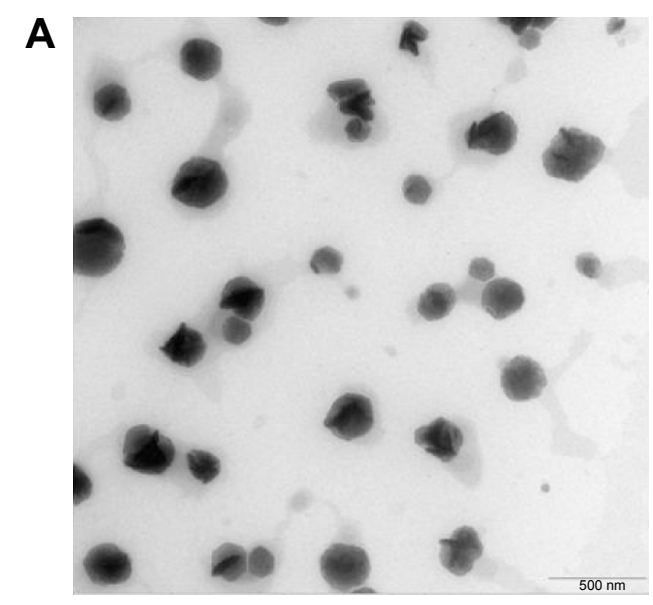

F1 8,000x

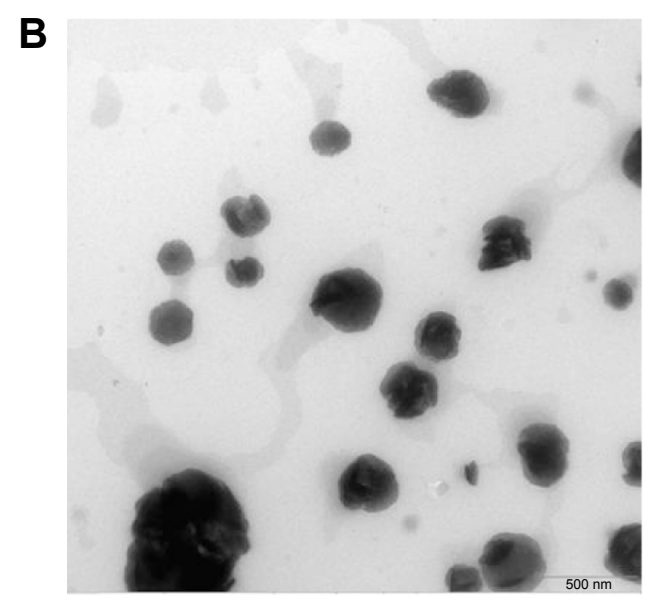

F2 8,000x

Figure 2 Transmission electron microscopy images of capsaicin-loaded cubosomes. Notes: (A) FI; (B) F2. Microscopy images were taken using a JEM I400 JEOL instrument (JEOL, Tokyo, Japan). Specimens were prepared by drop casting of cubosomes $(10 \mu \mathrm{L})$ on a copper grid with $10 \%$ phosphotungstic acid. The size distribution was determined by examining an overview transmission electron microscopy image using software.

11.5 software with one-way analysis of variance or Student's $t$-test. Differences were considered significant at $P<0.05$.

\section{Results and discussion}

\section{Particle size and morphology of capsaicin-loaded cubosomes}

The average particle size of capsaicin-loaded cubosomes F1 and F2 was $251 \mathrm{~nm}$ and $215 \mathrm{~nm}$, respectively, with a small polydispersity index $(<0.1)$, indicating homogeneous micelle size. Figure 2 showed the TEM images of capsaicin-loaded cubosomes, which demonstrated that the cubosomes of irregular hexagonal shape were well dispersed as individual particles. In addition, the particle size observed in Figure 2 was approximately $200 \mathrm{~nm}$, consistent with that determined by the particle detector.

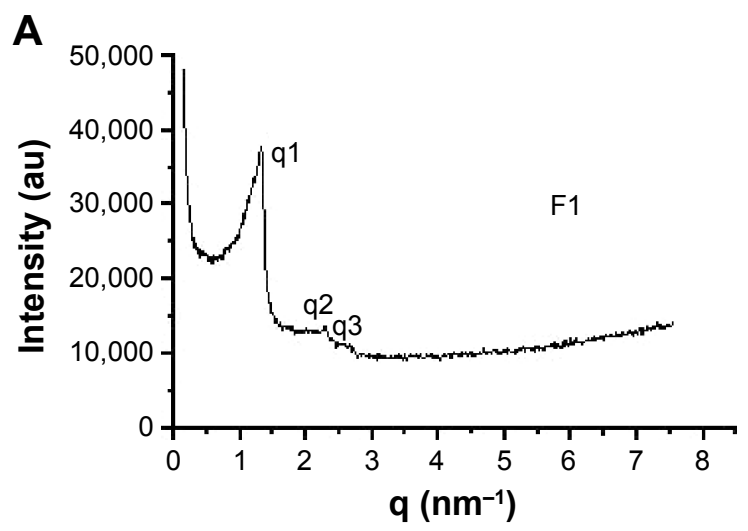

\section{SAXS}

The SAXS experiment was carried out to investigate the internal structure of the cubosome. The values of scattering vector $\mathrm{q}$ corresponding to the scattering peaks for cubosomes F1 and F2 (Figure 3) appeared in the ratio of $\mathrm{q} 1: \mathrm{q} 2: \mathrm{q} 3=\sqrt{2}: \sqrt{4}: \sqrt{6}$, which corresponded to the Miller indices, $\mathrm{hk} \ell=110, \mathrm{hk} \ell=200, \mathrm{hk} \ell=211$, respectively. The results fit the characteristic peaks of the Im3m crystallographic space group (Q229), which contains a primitive cube phase. ${ }^{16}$

\section{Encapsulation efficiency}

To determine the dialysis time of free drug, $2 \mathrm{~mL}$ of capsaicin solution was firstly sealed into the dialysis bag, and dialysis balance was reached within 4 hours (Figure 4). So, $2 \mathrm{~mL}$ of

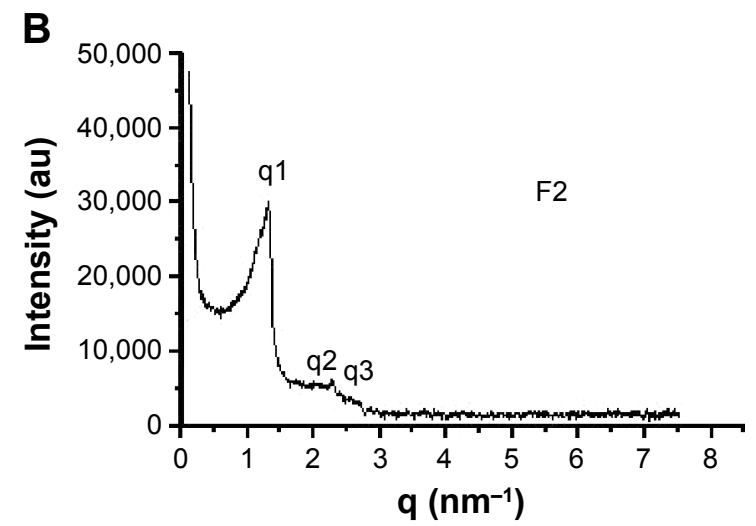

Figure 3 SAXS profiles of capsaicin-loaded cubosomes.

Notes: (A) FI; (B) F2. SAXS measurements were carried out on a high-flux SAXS instrument operating in the line of collimation and equipped with an imaging plate as a detector. The imaging plate with a pixel size of $42.3 \times 42.3 \mu \mathrm{m}^{2}$ was extended into a wide-angle range. The cubosome samples were carefully loaded into a quartz capillary with a diameter of $\mathrm{I} \mathrm{mm}$, and exposed for 60 minutes. Abbreviation: SAXS, small-angle X-ray scattering. 


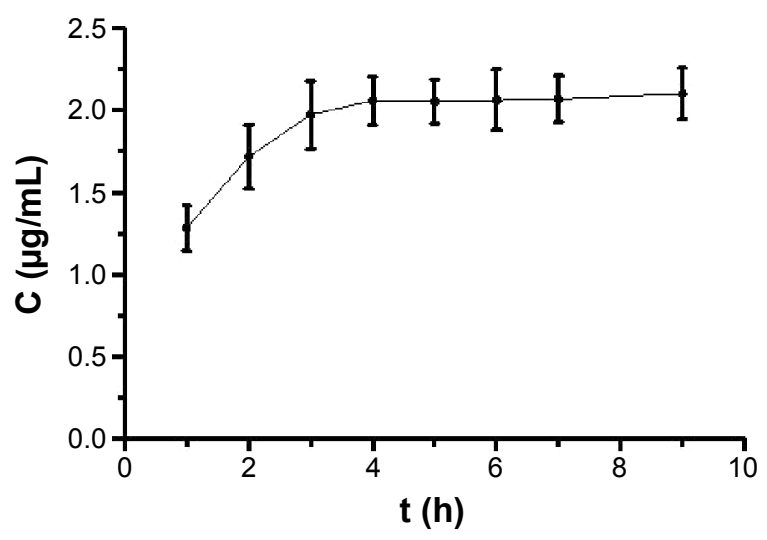

Figure 4 Dialysis curve of capsaicin solution $(n=3)$.

Notes: To determine the dialysis time, $2 \mathrm{~mL}$ of capsaicin solution was sealed into dialysis bags; the dialysis bags were placed into a flask and filled with $50 \mathrm{~mL}$ of water. The flask was then put in a rotary incubator shaker with a setting of $100 \mathrm{rpm}$ and $37^{\circ} \mathrm{C}$. The dialysis balance was reached within $4 \mathrm{~h}$.

Abbreviations: $\mathrm{C}$, concentration; $\mathrm{t}$, time; $\mathrm{h}$, hours; $\mathrm{n}$, number.

cubosome particles were placed in dialysis bags according to the process detailed earlier for 4 hours of testing. Calculated as the formula, the EEs of F1 and F2 were calculated as $97.14 \% \pm 0.54 \%$ and $97.58 \% \pm 0.53 \%$, respectively $(n=3)$. Such high EE could be attributed to the unique structure of the cubic phase and the property of capsaicin. Hydrophobic capsaicin can dissolve in GMO or PYT and distribute in the lipid bilayers. ${ }^{30}$

\section{In vitro release study}

The capsaicin release profiles from the cubosome particles (Figure 5) showed that the drug released faster from formulation F1 than from formulation F2, as about $41 \%$ and $33 \%$ of

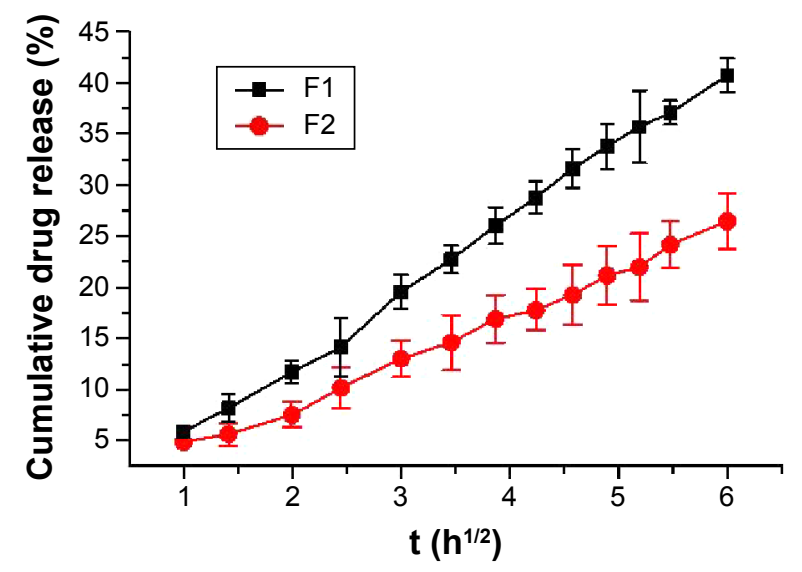

Figure 5 Cumulative release profiles of capsaicin from cubosomes $(n=3)$. Notes: A total of $5 \mathrm{~mL}$ of FI and F2 cubosomal samples were transferred into dialysis bags, which were placed into flasks and filled with $250 \mathrm{~mL}$ of water. The flasks were then put into a rotary incubator shaker at $50 \mathrm{rpm}$ and $37^{\circ} \mathrm{C}$. An aliquot of $5 \mathrm{~mL}$ was taken periodically and replaced with the same volume of water. The concentration of the released capsaicin was determined by HPLC on the basis of the standard curve. Abbreviations: t, time; h, hours; n, number; HPLC, high-performance liquid chromatography. capsaicin was released from F1 and F2 at 36 hours, respectively. Obviously, cubosomes could provide a sustained release system for capsaicin. Table 2 summarizes the results of fitting the release data for cubosomes. A linear relationship was found between capsaicin release and the square root of time $\left(R^{2}>0.99\right)$, indicating that the release is under diffusion control following Higuchi's equation. ${ }^{34}$

Though our previous study showed that the cubic phase gel also possessed diffusion-controlled sustained release properties with approximately $34 \%$ capsaicin released after 108 hours, ${ }^{30}$ the drug release rate from cubosomes was much faster than from the cubic phase gel. As cubosomes are discrete, submicron, or nanostructured particles formed from fragmentation of the cubic phase gels, their huge specific surface area provides more chances for capsaicin to diffuse from the lipid bilayers, resulting in faster release. ${ }^{30}$

\section{Comparison of permeation from cubosomes and cream}

The results of permeation experiments (Figure 6) showed that the permeation of capsaicin was linear with the permeating time for cubosomes and cream. The capsaicin permeation rates of F1, F2, and cream at steady state (Js) obtained from the slope of the linear portion of the cumulative amount that permeated through the rat skins per unit area versus time plot were calculated as follows: $0.32 \pm 0.05 \mu \mathrm{g} \cdot \mathrm{cm}^{-2}$.hour ${ }^{-1} ; 0.18 \pm 0.02 \mu \mathrm{g}$. $\mathrm{cm}^{-2} \cdot$ hour $^{-1}$; and $0.28 \pm 0.15 \mu \mathrm{g} \cdot \mathrm{cm}^{-2} \cdot$ hour $^{-1}$, respectively. Both PYT cubosomes and cream exhibited higher permeation $\left(0.32 \pm 0.05 \mu \mathrm{g} \cdot \mathrm{cm}^{-2} \cdot\right.$ hour $^{-1}$ and $0.28 \pm 0.15 \mu \mathrm{g} \cdot \mathrm{cm}^{-2} \cdot$ hour $\left.^{-1}\right)$ than did the GMO-cubosomes $\left(0.18 \pm 0.02 \mu \mathrm{g} \cdot \mathrm{cm}^{-2} \cdot\right.$ hour $\left.^{-1}\right)$.

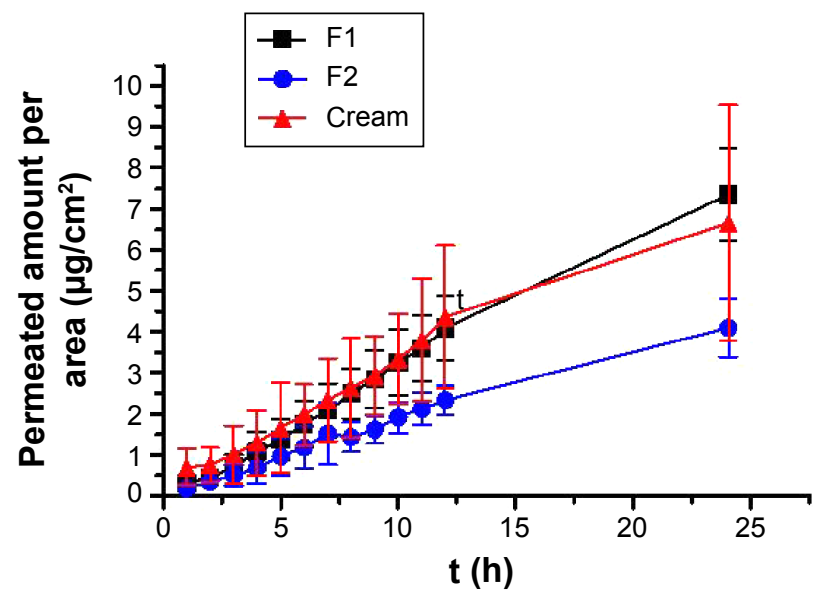

Figure 6 Permeation amount of capsaicin for different formulations $(n=3)$. Notes: In vitro permeation procedures of the capsaicin-contained cubosome application were determined using vertical Franz diffusion cells. The test formulations ( $\mathrm{I} \mathrm{g}$ in I mL) were applied on the diffusion surface of the skin $\left(2.8 \mathrm{~cm}^{2}\right.$ available diffusion area) in the donor compartment. The receiver compartment was filled with $6.5 \pm 0.5 \mathrm{~mL}$ of $20 \%$ ethanol solution, stirred at $300 \mathrm{rpm}$, and maintained at $32^{\circ} \mathrm{C} \pm 0.5^{\circ} \mathrm{C}$ using a circulating water bath. Skin permeation studies were performed for 24 hours. Abbreviations: $t$, time; $h$, hours; $n$, number. 


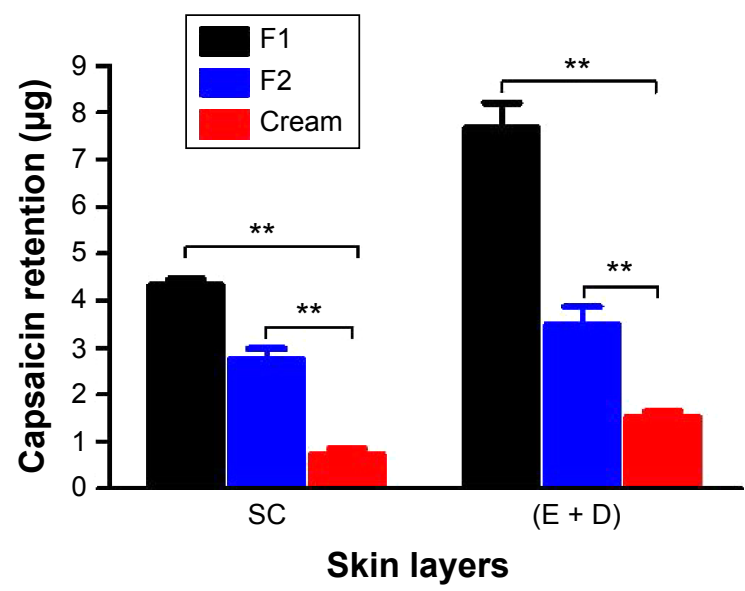

Figure 7 In vitro skin retention of capsaicin incorporated in different formulations. Notes: At 24 hours postapplication, the treated skin surfaces were thoroughly washed with distilled water and wiped with cotton swabs to remove excess formulation. SC and $(E+D)$ of the skin were separated by tape stripping. The capsaicin in both of these parts was extracted and analyzed using HPLC. $n=3$; $* * P<0.0$ l compared to the cream formulation.

Abbreviations: SC, stratum corneum; $(E+D)$, epidermis without stratum corneum + dermis; HPLC, high-performance liquid chromatography; n, number.

Furthermore, the permeation rate of capsaicin from the PYT cubosome $\left(0.32 \pm 0.05 \mu \mathrm{g} \cdot \mathrm{cm}^{-2} \cdot \mathrm{hour}^{-1}\right)$ was similar to that from cream $\left(0.28 \pm 0.15 \mu \mathrm{g} \cdot \mathrm{cm}^{-2} \cdot\right.$ hour $\left.^{-1}\right)$. Obviously, cubosomes formed from different liquid crystal materials possess significantly different permeation characteristics.

Lopes et $\mathrm{al}^{33}$ reported that the cubic phase formed by GMO showed enhancement in penetration and retention in the $\mathrm{SC}$ and $(\mathrm{E}+\mathrm{D})$ of cyclosporin A. Steluti et $\mathrm{al}^{35}$ also reported that GMO significantly increased the in vitro skin permeation/retention of 5-aminolevulinic acid as compared with the control without GMO. In addition, with respect to GMO, the permeation rate and retention of the drug increased with the increase of GMO in the preparation. But the permeation and retention decreased as the GMO concentration reached $30 \%$ and $20 \%$, respectively.

Figure 7 shows the retention of capsaicin in $\mathrm{SC}$ and $(\mathrm{E}+\mathrm{D})$. The incorporation of capsaicin in cubosomes led to an increase of drug skin retention 24 hours postapplication. For the capsaicin-loaded cream formulation, $0.72 \pm 0.13 \mu \mathrm{g}$ and $1.52 \pm 0.11 \mu \mathrm{g}$ of the drug was retained in SC and $(\mathrm{E}+\mathrm{D})$, respectively. For capsaicin-loaded cubosomes, the drug retention in $\mathrm{SC}$ and $(\mathrm{E}+\mathrm{D})$ was enhanced approximately four to six times $(P<0.01)$ and two to five times $(P<0.01)$, respectively. ${ }^{36}$ Contrary to previous reports, which suggested that cubosomes could enhance the percutaneous penetration of drugs, the cubosomes in this study reduced the skin absorption of capsaicin as compared to the conventional cream formulation. The amount of capsaicin absorbed from the GMO-based cubosomes into the skin after 24-hour application was less than that from the conventional cream. In spite of the reduced absorption, the cubosomal formulations still achieved higher and sustained skin retention of capsaicin as compared to the cream. This may be due to the fact that the cubosomal formulation could retard the diffusion of the drug from the skin to the systemic blood stream.

As shown in Figure 5, the release of capsaicin from F1 was faster than that from F2; meanwhile, F1 showed
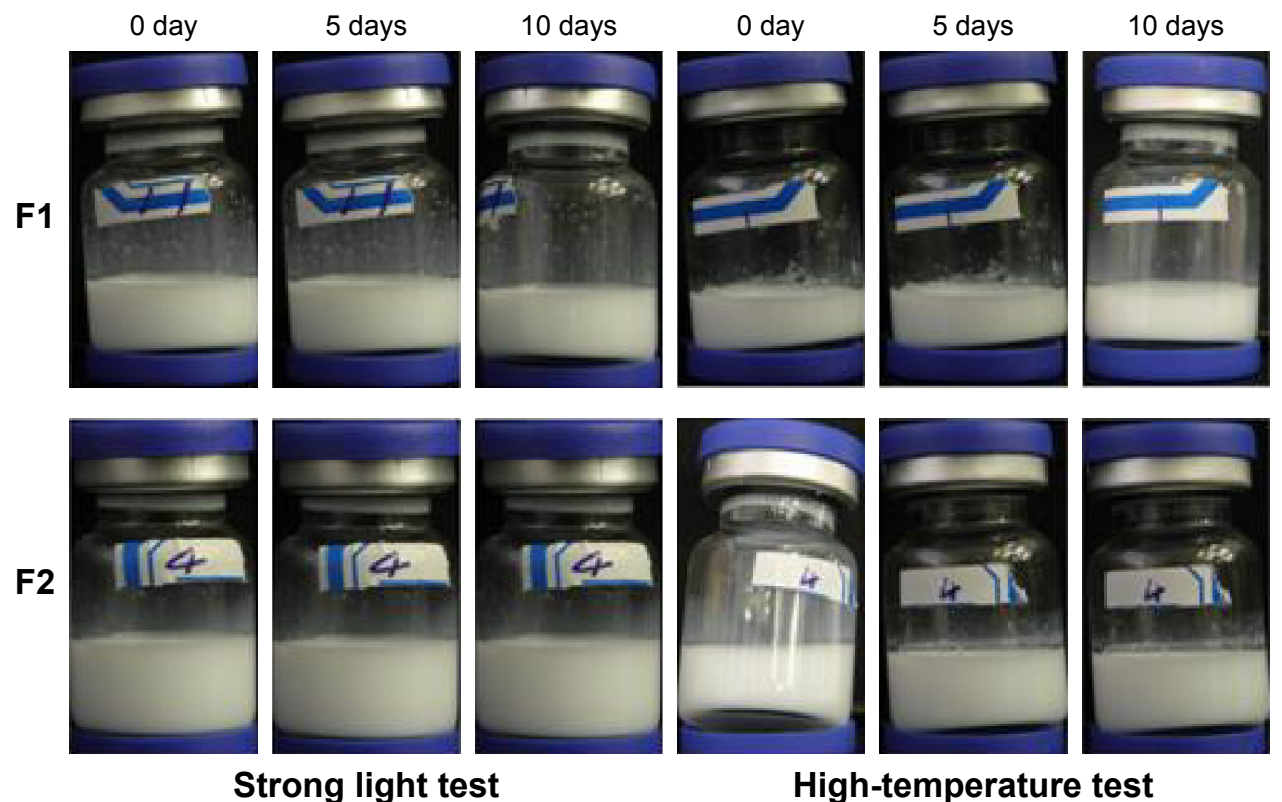

High-temperature test

Figure 8 Pictures of cubosomes before and after the stress test under strong light and high temperature $\left(60^{\circ} \mathrm{C}\right)$ for up to 10 days.

Notes: The stability study of the cubosomes was studied by stress testing. The cubosome samples were deposited and sealed in glass sterile vials, and then the vials were placed in a drug stability test chamber under strong light $(4,500 \pm 500 \mathrm{I} \times)$ and high temperature $\left(60^{\circ} \mathrm{C}\right)$, respectively, for 10 days. At the 5 th and 10 th day, the appearance and particle size of the samples were checked. 
Table 3 Particle size and size distribution of cubosomes under strong light and high-temperature stress test

\begin{tabular}{|c|c|c|c|c|c|}
\hline \multirow[t]{2}{*}{ Formulation } & \multirow[t]{2}{*}{ Time (days) } & \multicolumn{2}{|c|}{ Strong light $(4,500 \pm 500 \mathrm{I} \times)$} & \multicolumn{2}{|c|}{ High temperature $\left(60^{\circ} \mathrm{C}\right)$} \\
\hline & & Diameter $(\mathrm{nm})$ & PDI & Diameter $(\mathrm{nm})$ & PDI \\
\hline \multirow[t]{3}{*}{$\mathrm{FI}$} & 0 & 251.3 & 0.093 & 251.3 & 0.093 \\
\hline & 5 & 261 & 0.095 & 259.9 & 0.052 \\
\hline & 10 & 246.5 & 0.093 & 258.2 & 0.082 \\
\hline \multirow[t]{3}{*}{ F2 } & 0 & 215.7 & 0.099 & 215.7 & 0.099 \\
\hline & 5 & 228.5 & 0.097 & 229.1 & 0.078 \\
\hline & 10 & 253.2 & 0.162 & 227.7 & 0.117 \\
\hline
\end{tabular}

Notes: FI, PYT based cubosomes; F2, GMO based cubosomes. The concentration of capsaicin in both cubosomes are $250 \mu \mathrm{g} / \mathrm{mL}$.

Abbreviations: PDI, polydispersity index; PYT, phytantriol; GMO, glycerol monooleate.

higher drug permeation and retention than F2, and the drug permeation was positive for the drug release.

\section{Stability results}

Either under strong light or a high temperature of $60^{\circ} \mathrm{C}$ for 10 days, the cubosome particles were maintained as a milkwhite emulsion without obvious aggregation (Figure 8). Table 3 shows that the particle sizes of cubosomes remained stable during the 10-day stress test. Therefore, the stress test demonstrated that the cubosomes comprised a thermodynamically stable system.

\section{Evaluation of skin irritation}

The images of skin tissues treated with cubosomes and cream formulations are shown in Figure 9. The skin structure was clear and integral without inflammatory reaction or infiltration
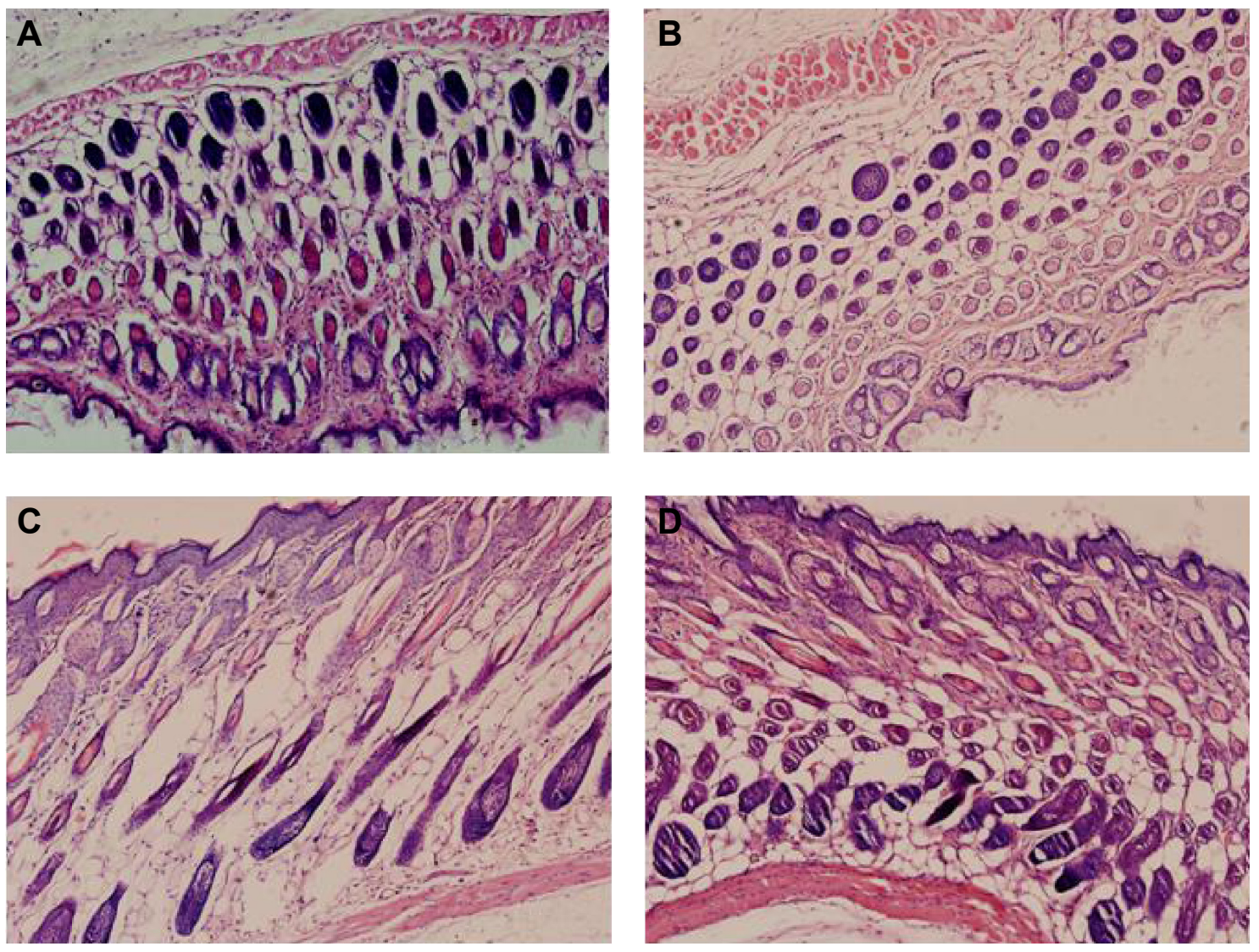

Figure 9 Microscopic photographs of rat skin after treatment of cubosomes and cream $(\times 100)$.

Notes: (A) Control; (B) cream; (C) FI; and (D) F2. Capsaicin formulations were applied topically on a limited area $\left(2 \times 2 \mathrm{~cm}^{2}\right)$ of skin on the back of a mouse twice a day for I week. On the 7th day, the mice were killed and the treated skin area was dissected. After fixation, the skin samples were dehydrated and embedded in paraffin, and then $5 \mu \mathrm{m}$ sections of skin were stained with hematoxylin and eosin for observation under microscope. The skin of the untreated mouse was used as the control. 
of the inflammatory cells. Also, the skin treated with the cubosomes and cream showed very similar characteristics as that of the normal skin. These results demonstrated that both cubosomes and cream formulations had no obvious irritation to the skin.

\section{Conclusion}

The prepared cubosomes provided sustained release of capsaicin under diffusion control. Though the percutaneous absorption of capsaicin from the GMO-based cubosomes was less than that from the conventional cream, cubosomes still yielded higher and sustained skin retention of the drug than did the cream due to the retarded diffusion of the absorbed drug in the skin. Certainly, the mechanism of the retarded diffusion needs to be further investigated. The skin-targeted, sustained, and thermodynamically stable characteristics of cubosomal particles provide an interesting system for the topical delivery of capsaicin for alleviating postincision pain.

\section{Acknowledgments}

The authors gratefully acknowledge the financial support for the research (numbers: 81001643; 81173002; 81202477; 2013B021800073; 2CY13002; 2014108101049 and 201450715200682). This work was also supported in part by China Scholarship Council (numbers: 201308440229 and 201408440141). We further acknowledge Danisco Company, Denmark, for the generous gift samples of GMO.

\section{Disclosure}

The authors report no conflicts of interest in this work.

\section{References}

1. Huang XF, Xue JY, Jiang AQ, Zhu HL. Capsaicin and its analogues: structure-activity relationship study. Curr Med Chem. 2013;20(21): 2661-2672.

2. Sharma SK, Vij AS, Sharma M. Mechanisms and clinical uses of capsaicin. Eur J Pharmacol. 2013;720(1-3):55-62.

3. Rollyson WD, Stover CA, Brown KC, et al. Bioavailability of capsaicin and its implications for drug delivery. J Control Release. 2014; 196:96-105.

4. Wohlrab J, Neubert RH, Heskamp ML, Michael J. Cutaneous drug delivery of capsaicin after in vitro administration of the $8 \%$ capsaicin dermal patch system. Skin Pharmacol Physiol. 2015;28(2):65-74.

5. Sarwa KK, Mazumder B, Rudrapal M, Verma VK. Potential of capsaicin-loaded transfersomes in arthritic rats. Drug Deliv. Epub 2014 Jan 29.

6. Kumar Sarwa K, Rudrapal M, Mazumder B. Topical ethosomal capsaicin attenuates edema and nociception in arthritic rats. Drug Deliv. Epub 2014 Feb 10.

7. Zhu Y, Wang M, Zhang J, et al. Improved oral bioavailability of capsaicin via liposomal nanoformulation: preparation, in vitro drug release and pharmacokinetics in rats. Arch Pharm Res. 2015;38(4):512-521.
8. Somagoni J, Boakye CH, Godugu C, et al. Nanomiemgel - a novel drug delivery system for topical application - in vitro and in vivo evaluation. PLoS One. 2014;9(12):e115952.

9. Gupta R, Gupta M, Mangal S, Agrawal U, Vyas SP. Capsaicin-loaded vesicular systems designed for enhancing localized delivery for psoriasis therapy. Artif Cells Nanomed Biotechnol. 2014:1-10.

10. Schoellhammer CM, Blankschtein D, Langer R. Skin permeabilization for transdermal drug delivery: recent advances and future prospects. Expert Opin Drug Deliv. 2014;11(3):393-407.

11. Jain A, Jain P, Kurmi J, et al. Novel strategies for effective transdermal drug delivery: a review. Crit Rev Ther Drug Carrier Syst. 2014;31(3): 219-272.

12. Mitragotri S, Burke PA, Langer R. Overcoming the challenges in administering biopharmaceuticals: formulation and delivery strategies. Nat Rev Drug Discov. 2014;13(9):655-672.

13. Lee KW, Nguyen TH, Hanley T, Boyd BJ. Nanostructure of liquid crystalline matrix determines in vitro sustained release and in vivo oral absorption kinetics for hydrophilic model drugs. Int J Pharm. 2009; 365(1-2):190-199.

14. Caffrey M. A lipid's eye view of membrane protein crystallization in mesophases. Curr Opin Struct Biol. 2000;10(4):486-497.

15. Shah JC, Sadhale Y, Chilukuri DM. Cubic phase gels as drug delivery systems. Adv Drug Deliv Rev. 2001;47(2-3):229-250.

16. Pan X, Han K, Peng X, et al. Nanostructured cubosomes as advanced drug delivery system. Curr Pharm Des. 2013;19(35):6290-6297.

17. Bender J, Ericson MB, Merclin N, et al. Lipid cubic phases for improved topical drug delivery in photodynamic therapy. J Control Release. 2005; 106(3):350-360.

18. Chang CM, Bodmeier R. Low viscosity monoglyceride-based drug delivery systems transforming into a highly viscous cubic phase. Int $J$ Pharm. 1998;173(1-2):51-60.

19. Wadsten-Hindrichsen P, Bender J, Unga J, Engström S. Aqueous self-assembly of phytantriol in ternary systems: effect of monoolein, distearoylphosphatidylglycerol and three water-miscible solvents. J Colloid Interface Sci. 2007;315(2):701-713.

20. Spicer PT, Hayden KL, Lynch ML, Ofori-Boateng A, Burns JL. Novel process for producing cubic liquid crystalline nanoparticles (cubosomes). Langmuir. 2001;17(19):5748-5756.

21. Barauskas J, Johnsson M, Joabsson F, Tiberg F. Cubic phase nanoparticles (Cubosome): principles for controlling size, structure, and stability. Langmuir. 2005;21(6):2569-2577.

22. Salentinig S, Yaghmur A, Guillot S, Glatter O. Preparation of highly concentrated nanostructured dispersions of controlled size. J Colloid Interface Sci. 2008;326(1):211-220.

23. Kim MK, Chung SJ, Lee MH, Cho AR, Shim CK. Targeted and sustained delivery of hydrocortisone to normal and stratum corneumremoved skin without enhanced skin absorption using a liposome gel. J Control Release. 1997;46(3):243-251.

24. Gan L, Han S, Shen J, et al. Self-assembled liquid crystalline nanoparticles as a novel ophthalmic delivery system for dexamethasone: Improving preocular retention and ocular bioavailability. Int J Pharm. 2010;396(1-2):179-187.

25. Morsi NM, Abdelbary GA, Ahmed MA. Silver sulfadiazine based cubosome hydrogels for topical treatment of burns: development and in vitro/in vivo characterization. Eur J Pharm Biopharm. 2014;86(2): 178-189.

26. Yang Z, Peng X, Tan Y, et al. Optimization of the preparation process of an oral phytantriol-based amphotericin B cubosomes. J Nanomater. 2011;(2011):1-10.

27. Peng XS, Zhou YF, Han K, Qin LZ, Wu CB. [Preparation and in vitro study on diffusion of capsaicin cubosome]. Zhongguo Zhong Yao Za Zhi. 2014;39(4):644-647. Chinese.

28. Peng X, Yang Z, Chen M, Han K, Xiao Y, Wu C. [Preparation, characterization and content determination of cubic phase gel containing capsaicin]. Zhongguo Zhong Yao Za Zhi. 2010;35(23):3123-3126. Chinese. 
29. Liu H, Pan WS, Nie SF, Yang XG, Yan TX. [Study on in vitro release empirical method and the release mechanism of budesonide colonic localization tablet]. Yaо Хие Хие Ваo. 2008;43(11):1147-1151. Chinese.

30. Peng X, Wen X, Pan X, Wang R, Chen B, Wu C. Design and in vitro evaluation of capsaicin transdermal controlled release cubic phase gels. AAPS Pharm Sci Tech. 2010;11(3):1405-1410.

31. Rottke M, Lunter DJ, Daniels R. In vitro studies on release and skin permeation of nonivamide from novel oil-in-oil-emulsions. Eur J Pharm Biopharm. 2014;86(2):260-266.

32. Zhang $\mathrm{P}, \mathrm{Gao} \mathrm{W}$, Zhang L, et al. In vitro evaluation of topical microemulsion of capsaicin free of surfactant. Biol Pharm Bull. 2008;31(12): 2316-2320.

33. Lopes LB, Lopes JL, Oliveira DC, et al. Liquid crystalline phases of monoolein and water for topical delivery of cyclosporin A: characterization and study of in vitro and in vivo delivery. Eur J Pharm Biopharm. 2006;63(2):146-155.
34. Tanaka N, Imai K, Okimoto K, et al. Development of novel sustainedrelease system, disintegration-controlled matrix tablet (DCMT) with solid dispersion granules of nilvadipine. J Control Release. 2005; 108(2-3):386-395.

35. Steluti R, De Rosa FS, Collett J, Tedesco AC, Bentley MV. Topical glycerol monooleate/propylene glycol formulations enhance 5 -aminolevulinic acid in vitro skin delivery and in vivo protophorphyrin IX accumulation in hairless mouse skin. Eur J Pharm Biopharm. 2005;60(3):439-444.

36. Kwon TK, Hong SK, Kim J-C. In vitro skin permeation of cubosomes containing triclosan. Journal of Industrial and Engineering Chemistry. 2012;18(1):563-567.

\section{Publish your work in this journal}

Drug Design, Development and Therapy is an international, peerreviewed open-access journal that spans the spectrum of drug design and development through to clinical applications. Clinical outcomes, patient safety, and programs for the development and effective, safe, and sustained use of medicines are a feature of the journal, which has also been accepted for indexing on PubMed Central. The manuscript management system is completely online and includes a very quick and fair peer-review system, which is all easy to use. Visit http://www.dovepress.com/testimonials.php to read real quotes from published authors.

Submit your manuscript here: http://www.dovepress.com/drug-design-development-and-therapy-journal 